\title{
NÍVEIS DE ANSIEDADE COMPETITIVA EM JOGADORES DE VOLEIBOL DO INTERIOR DO PARANÁ
}

\author{
LEVELS OF COMPETITIVE ANXIETY IN VOLLEYBALL PLAYERS OF THE CONUTRYSIDE OF PARANÁ
}

\author{
Veronica Volski ${ }^{*}$, Sivano da Silva Coutinho ${ }^{b^{*}}$, Sheila Aparecida de Souza ${ }^{\mathrm{c}^{*}}$ \\ avero_edf@hotmail.com, bsilvano.unicentro@gmail.com, csheila.souza28@yahoo.com \\ *Universidade Estadual do Centro-Oeste - Guarapuava (PR), Brasil.
}

Data de entrada do artigo: 02/12/2013

Data de aceite do artigo: 05/05/2014

\section{RESUMO}

Introduçáo: A ansiedade é um estado que se caracteriza por sensações de risco impendente, associada a uma atitude de espera, e que provoca uma inquietaçáo mais ou menos profunda na personalidade do indivíduo, em diversos momentos na vida e em diversos níveis, podendo ser prejudicial ou benéfica. No esporte, a ansiedade em excesso pode ser considerada um fator negativo. Objetivos: Avaliar os níveis de ansiedade-traço e ansiedade-estado de jogadores de voleibol das categorias infantil e infanto-juvenil de Guarapuava, bem como comparar tais níveis entre os sexos e as categorias. Materiais e métodos: A amostra foi composta por 34 sujeitos do sexo masculino e feminino com idade entre 13 e 16 anos. Os instrumentos das coletas de dados foram o questionário Sport Competition Anxiety Test (SCAT) e o questionário Competitive State Anxiety Inventory-2 (CSAI-2). Resultados: Os resultados encontrados foram de níveis médios de ansiedade-traço em todas as categorias. A ansiedade-estado cognitiva apresentou valores baixos para a categoria infantil masculino e infanto-juvenil, porém níveis elevados para a categoria infantil feminino. Para a ansiedade-estado somática, os valores foram todos baixos e, para o fator autoconfiança, foram todos elevados, em todas as categorias. Conclusóes: A partir dos resultados encontrados, pode-se concluir que não há diferenças significativas nos níveis de ansiedade se comparados entre os gêneros, ainda que todas as categorias apresentassem níveis baixos para ansiedade cognitiva. Isso mostra que os atletas se mostraram seguros com a competiçáo.

Palavras-chave: ansiedade; voleibol; competição.

\section{ABSTRACT}

Introduction: Anxiety is a state characterized by feelings of impending risk associated with a waiting attitude, and it causes a more or less deep concern in the personality of the individual, at various times in life and at different levels, which may be harmful or beneficial. In sports, anxiety in excess can be considered a negative factor. Objetives: To assess the levels of trait anxiety and state anxiety of volleyball players of youth leagues in Guarapuava and to compare these levels between genders and leagues. Materials and Methods: The sample consisted of 34 male and female subjects aged between 13 to 16 years. The instruments of data collection were the questionnaire Sport Competition Anxiety Test - SCAT, and the questionnaire Competitive State Anxiety Inventory - 2 (CSAI - 2). Results: The results showed average levels of trait anxiety in all leagues. Cognitive state anxiety showed low values for male and juvenile child leagues, but high levels for infant female leagues. For somatic state anxiety the values were low and the confidence factor was high in all leagues. Conclusions: Through the results it can be concluded that there are no significant differences in anxiety levels between genders, although all leagues presented low levels for cognitive anxiety. This shows that athletes are self-confident regarding to competition.

Keywords: Anxiety, Volleyball, Competition. 


\section{Introdução}

A ansiedade configura-se como um estado psicológico presente em diversos momentos do cotidiano dos indivíduos. Ela pode se apresentar em diferentes níveis e ser considerada prejudicial ou benéfica. Considerada como uma experiência particular de cada indivíduo, de acordo com a personalidade deste, pode levá-lo a se sentir em situação de perigo, de aflição, entre outros.

Caracteriza-se por uma sensação de risco impendente, associada a uma atitude de espera, e que provoca uma inquietaçáo mais ou menos profunda na personalidade do indivíduo ${ }^{1}$. A ansiedade é considerada um estado emocional negativo no qual sentimentos de inquietação, preocupação e nervosismo estão coligados com energização ou perturbação do corpo. Uma pessoa ansiosa apresenta diversos sintomas, que interferem em suas estratégias de resolver determinada situação ${ }^{2}$.

Assim sendo, a ansiedade caracteriza-se por sentimentos de emoção, como preocupação e tensão, levando o indivíduo a sofrimentos antecipados, pressentimentos e crises ${ }^{3,4}$. Trata-se de uma condiçáo de alerta do organismo que causa sentimento de insegurança e pode comprometer o plano psicológico e físico ${ }^{6}$.

Ela pode ser dividida em ansiedade-traço e ansiedade-estado. A primeira refere-se a uma tendência comportamental, faz parte da personalidade do indivíduo e é relativamente estável. Apresenta-se como uma disposição de comportamento em compreender de forma ameaçadora as situaçóes que normalmente não são perigosas e em reagir a elas com ansiedade-estado. As pessoas que apresentam ansiedade-traço elevada geralmente possuem ansiedade-estado superior em situaçóes avaliativas e competitivas ${ }^{2}$.

Por sua vez, a ansiedade-estado refere-se ao estado comportamental rápido e temporário, que pode estar sempre em variação; assim, em determinados momentos, um indivíduo pode se sentir mais ansioso do que em outros, principalmente nos momentos de tensão e apreensão, e a ansiedade varia de intensidade conforme a ocasião ${ }^{6}$. É uma predisposição em ocasiôes de nervosismo e perigo ${ }^{7}$.

A ansiedade-estado subdivide-se em cognitiva, somática e autoconfiança. A ansiedade-estado cognitiva refere-se ao quanto uma pessoa se preocupa ou tem pensamentos negativos em relação a alguma situação. Já a ansiedade-estado somática diz respeito às alteraçóes na ativação fisiológica em diferentes momentos. A autoconfiança define-se por diferença pessoal que conglomera a percepção de confiança do indivíduo, auxiliando -o a realizar com êxito suas atividades ${ }^{8,9}$.

No âmbito esportivo, a ansiedade excessiva pode ser considerada um fator negativo, visto que pode afetar o desempenho do atleta. Contudo, os níveis de ansiedade podem variar de atleta para atleta conforme a personalidade, o tempo de prática em determinada modalidade, a idade, o sexo, entre outros fatores ${ }^{10}$.

Além do treinamento físico e tático, os atletas necessitam de um treinamento psicológico. Muitos, considerados melhores jogadores durante o treino, podem não render durante uma competição o quanto o técnico e os companheiros de time acreditavam que renderiam. Nesse sentido, são comuns situaçóes em que, durante os treinos, os atletas apresentam menores níveis de ansiedade do que em competição ${ }^{11}$.

Uma vez que a sociedade em que vivemos valoriza apenas a vitória e teme o fracasso ${ }^{12}$, essa condição faz, de certa forma, que os níveis de ansiedade dos atletas se elevem durante uma situaçáo competitiva, levando seu desempenho a cair. Por isso, o treinamento psicológico é visto como um fator importante, já que ajuda o atleta a aprender a lidar com essas situações e, principalmente, a controlar os seus níveis de ansiedade.

O voleibol é uma das modalidades esportivas mais praticadas no Brasil e no mundo. Embora seja um esporte que náo exige o contato físico direto com o adversário, isso não significa que o atleta de voleibol não deve desenvolver um planejamento técnico e tático com a finalidade de ultrapassar os sistemas táticos elaborados pelo adversário. E, assim como em outras modalidades, esse fato pode gerar níveis de ansiedade nos atletas no momento em que executam tal planejamento, seja no saque, na recepçáa, no levantamento, no ataque, no bloqueio, na defesa, entre outros.

Entretanto, são recentes os estudos que visam traçar perfis psicológicos de atletas nas diversas modalidades, inclusive no voleibol ${ }^{5,10,13,14,15,16,17}$.

Tendo em vista que é determinante no desempenho do atleta, que pode ou não afetá-lo, o fator psicológico que será estudado é a ansiedade (traço e estado). Para tanto, este estudo busca avaliar e comparar os níveis de ansiedade-traço e estado entre as categorias e entre os gêneros dos jogadores de voleibol das categorias infantil e infanto-juvenil de Guarapuava.

\section{Metodologia}

\section{Amostra}

A amostra foi composta por 34 sujeitos do sexo feminino e masculino, com idade entre 13 e 16 anos. Os atletas foram divididos em: categoria infantil feminino (12 atletas); categoria infantil masculino (11 atletas); e categoria infanto-juvenil feminino (11 atletas). Todos os atletas são participantes de equipes de voleibol da cidade de Guarapuava, estado do Paraná, e treinam 
no Grêmio dos Subtenentes e Sargentos da Guarnição de Guarapuava (Gresga) e no Ginásio Municipal de Esportes Santa Terezinha. Foram excluídos do estudo os atletas que náo responderem a todas as perguntas de qualquer um dos questionários, os atletas que não compareceram ao treino no dia de aplicaçáo do questionário SCAT e os atletas que náo competiram e não responderam ao questionário CSAI-2.

\section{Instrumentos de Medida}

Para avaliar a ansiedade-traço, foi utilizada a Escala de Ansiedade-traço Competitiva (Sport Competition Anxiety Test, ou SCAT) ${ }^{18}$, composta por 15 questóes indicadoras de ansiedade-traço competitiva. Essas 15 questôes estão distribuídas em 8 itens de ativação $(2,3$, $5,8,9,12,14$ e 15), 2 itens de desativação $(6$ e 11) e 5 itens que possuem efeito placebo e não são contados ( 1 , $4,7,10,13)$ e são respondidas numa escala tipo Likert de 3 pontos, em que 1 = dificilmente; 2 = às vezes; $3=$ sempre. A pontuação é dada de 1 a 3 para os itens de ativação e de 3 a 1 para os de desativaçáo. $\mathrm{O}$ escore total do teste varia de 10 (baixa ansiedade-traço) a 30 (alta ansiedade-traço).

Para avaliar a ansiedade-estado, foi utilizada a Escala de Ansiedade-estado (Competitive State Anxiety Inventory-2, ou CSAI-2) ${ }^{8}$, composta por 27 questôes, sendo estas divididas em 9 questóes que avaliam a ansiedade cognitiva $(1,4,7,10,13,16,19,22$ e 25$), 9$ que avaliam a ansiedade somática $(2,5,8,11,14,17$, 20,23 e 26) e outras 9 que avaliam a autoconfiança (3, $6,9,12,15,18,21,24$ e 27). As questôes são respondidas em uma escala tipo Likert de 4 pontos, em que 1 = um pouco; 2 = razoável; 3 = moderado $4=$ muito. A classificação de 9 a 18 pontos indica baixos níveis de ansiedade-estado; de 19 a 27, níveis moderados; e de 28 a 36, níveis elevados.

\section{Procedimentos da Coleta de Dados}

Após a aceitação do convite e autorização por parte dos técnicos de voleibol das categorias infantil feminino e masculino e infanto-juvenil feminino de Guarapuava e a aprovação no Comitê de Ética pelo parecer $n^{\circ}$ 292.988/2013, iniciou-se a coleta dos dados. Primeiro, os atletas, visto que eram menores de idade, levaram o Termo de Consentimento Livre e Esclarecido (TCLE) para assinatura dos responsáveis. No dia seguinte de treinamento, os atletas responderam ao questionário SCAT, cada categoria em seu local de treinamento. O questionário CSAI-2 foi respondido na primeira etapa da Liga de Voleibol do Paraná, trinta minutos antes do primeiro jogo de cada categoria.

\section{Procedimentos Estatísticos}

Para tabular os dados, foi utilizado o Microsoft Office Excel 2007 e, para a análise estatística, foi utilizado o programa SPSS ${ }^{\circ} 13.0$. Os resultados foram representados como média e desvio-padrão. O teste ANOVA One-Way foi utilizado para verificar se havia diferença significativa entre os grupos. Os valores foram considerados estatisticamente significativos somente para $\mathrm{p}<$ 0,05. Quando apropriado, foi utilizado o teste post hoc de Tukey para identificar as diferenças entre grupos.

\section{Resultados}

\section{Caracterização da Amostra}

A Tabela 1 apresenta a caracterização da amostra estudada (atletas de voleibol de Guarapuava), composta por 34 sujeitos, do sexo masculino e feminino, das categorias infantil (feminino e masculino) e infanto-juvenil (feminino). A categoria infanto-juvenil masculina não fez parte deste estudo, pois não havia atletas suficientes para a formação de uma equipe para a competição.

Tabela 1: Caracterização da amostra composta por 34 sujeitos, atletas de voleibol de Guarapuava.

\begin{tabular}{ccc}
\hline Categorias & Média de idade (anos) & $\begin{array}{c}\text { Média de tempo } \\
\text { de prática (anos) }\end{array}$ \\
\hline Infantil F & 14 & 4,27 \\
Infantil M & 13,41 & 3,91 \\
Infanto-juvenil F & 15,90 & 6 \\
\hline
\end{tabular}

A Tabela 2 apresenta os níveis de ansiedade-traço, obtidos a partir do questionário SCAT, e os compara entre as categorias. Pode-se perceber que não há diferenças estatisticamente significativas entre as categorias

Tabela 2: Análise dos níveis de ansiedade entre as categorias e entre os gêneros, a partir do questionário SCAT.

\begin{tabular}{cc}
\hline Grupo & $\begin{array}{c}\text { Nível de ansiedade } \\
\text { (média } \pm \text { DP) }\end{array}$ \\
\hline Infantil M & $19,36 \pm 3$ \\
Intantil F & $20,66 \pm 2$ \\
Infanto-Juvenil F & $20,54 \pm 3$ \\
\hline
\end{tabular}

A Tabela 3 apresenta a frequência de respostas, por categoria, em cada classificação de níveis de ansiedade-traço. 
Tabela 3: Frequência absoluta e relativa da ansiedade-traço, separada por categorias.

\begin{tabular}{ccccccc}
\hline $\begin{array}{c}\text { Classificaçáo da } \\
\text { ansiedade-traço }\end{array}$ & $\begin{array}{c}\text { Infantil } \\
\text { feminino }\end{array}$ & \multicolumn{2}{c}{$\begin{array}{c}\text { Infantil } \\
\text { masculino }\end{array}$} & $\begin{array}{c}\text { Infanto- } \\
\text {-juvenil } \\
\text { feminino }\end{array}$ \\
\hline Baixa & $f$ & $\%$ & $f$ & $\%$ & $f$ & $\%$ \\
Média-baixa & 0 & 0 & 0 & 0 & 0 & 0 \\
Média & 12 & 100 & 1 & 9,09 & 1 & 9,09 \\
Média-alta & 0 & 0 & 2 & 18,18 & 1 & 9,09 \\
Alta & 0 & 0 & 0 & 0 & 0 & 0 \\
\hline
\end{tabular}

$\mathrm{Na}$ categoria infantil feminino, todas as jogadoras se enquadraram no nível médio. Na categoria infantil masculino, observa-se que apenas 1 pessoa $(9,09 \%)$ apresentou nível médio-baixo de ansiedade-traço, 8 pessoas $(72,72 \%)$ apresentaram nível médio com maior frequência, enquanto 2 pessoas $(18,18 \%)$ se enquadraram no nível de ansiedade-traço médio-alto. A categoria infanto-juvenil feminino também apresentou maior frequência no nível de ansiedade média, com 9 pessoas $(81,81 \%)$; 1 pessoa $(9,09 \%)$ apresentou nível médiobaixo e 1 apresenta nível médio-alto.

A Figura 1 apresenta os níveis de ansiedade-estado cognitiva, somática e autoconfiança, comparando-os entre as categorias.

Figura 1: Análise, entre as categorias, dos níveis de ansiedade (A) cognitiva, (B) somático e (C) autoconfiança a partir do questionário CSAI-2.

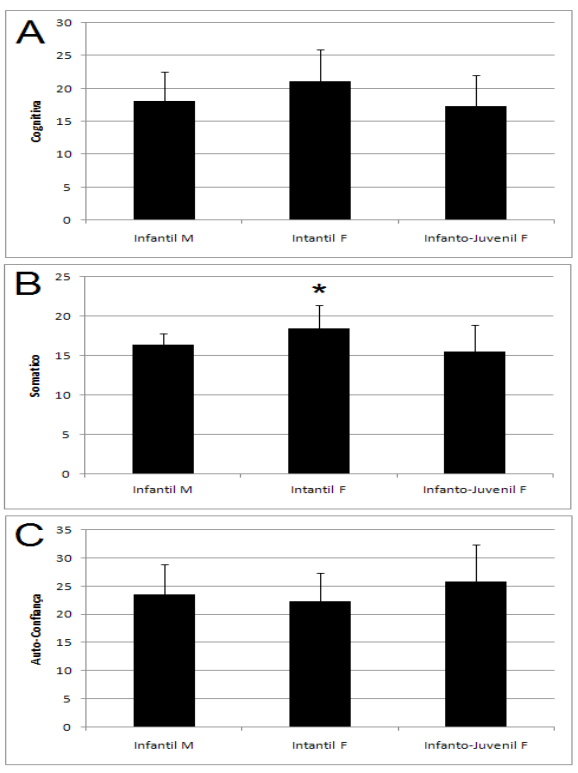

Os dados são apresentados em média e desvio-padrão ${ }^{*} \mathrm{p}<0,05$, estatisticamente significante em relação aos grupos infanto-juvenil feminino e infantil feminino (ANOVA One Way após post hoc de Tukey, $\mathrm{p}<0,05$ ).
O painel A mostra os níveis de ansiedade cognitiva entre as categorias. Os resultados não apresentam diferenças estatisticamente significativas. Observa-se também que todos os atletas apresentam níveis moderados, segundo a classificaçáo da ansiedade-estado.

O painel B apresenta a diferença nos níveis de ansiedade somática entre as categorias. Os resultados mostram uma diferença estatisticamente significante entre as categorias infantil feminino e infanto-juvenil feminino; os níveis da equipe infantil foram superiores aos da equipe infanto-juvenil. Pode-se notar que, na classificação dos níveis de ansiedade-estado somática, todas as categorias encontram-se em níveis baixos.

Por último, o painel $\mathrm{C}$ exibe a média dos níveis de autoconfiança entre as categorias. As diferenças observadas não são estatisticamente significativas. Em todas as categorias, os resultados se enquadram em níveis moderados de autoconfiança, segundo a classificação de ansiedade-estado.

\section{Discussão}

Os resultados apresentados na Tabela 2 náo corroboram o estudo realizado com nadadores amadores que comparou as médias na escala SCAT e as confrontou entre categorias; neste, nadadores mais jovens (categoria infantil) apresentaram maiores níveis de ansiedade-traço competitiva do que nadadores mais velhos (categoria sênior $)^{17}$.

Isso pode ser explicado pelo fato de que é nos primeiros anos da juventude que os níveis de ansiedade são mais elevados, geralmente por volta dos 14 aos 18 $\operatorname{anos}^{19}$. Com base nisso, sabe-se que os níveis de ansiedade aumentam no final da adolescência, tendendo a diminuir a partir dos 30 anos e podendo se elevar novamente a partir dos 60 anos ${ }^{14}$. Dadas as médias de idade dos atletas de voleibol analisados no presente estudo, essas afirmaçóes apoiam os resultados dos níveis de ansiedade-traço encontrados a partir do questionário SCAT.

Ainda analisando a Tabela 2, pode-se notar que não há uma diferença estatisticamente significativa entre os níveis de ansiedade-traço com relação ao gênero. Os resultados encontrados no presente estudo com relação à diferença nos níveis de ansiedade-traço entre os gêneros contradizem os achados do mencionado estudo com nadadores, em que atletas do sexo feminino apresentaram níveis de ansiedade-traço e estado mais elevados do que atletas do sexo masculino ${ }^{17}$.

Entretanto, os achados do presente estudo com relação ao gênero corroboram o estudo que buscou investigar os níveis de ansiedade competitiva de adolescentes e comparar entre os gêneros, a maturação, o nível de 
experiência e as modalidades esportivas e não encontrou diferenças significativas nos níveis de ansiedade entre adolescentes do sexo feminino e masculino ${ }^{20}$.

Com base na Tabela 3, pode-se observar que todos os atletas apresentaram maior frequência no nível de ansiedade médio; esse achado corrobora a literatura que ressalva que atividades mais complexas, como, por exemplo, os jogos pré-desportivos, requerem níveis de ansiedade menos elevados para o bom desempenho das funçóes ${ }^{21,14}$. Ressalta-se também que esportes coletivos geram menores níveis de ansiedade em comparação a esportes individuais, uma vez que, nestes últimos, o atleta não tem com quem dividir as responsabilidades, ficando mais exposto a julgamentos dos expectadores ${ }^{22}$.

O nível médio de ansiedade-traço encontrado com maior frequência na categoria infantil masculino corrobora o estudo que avaliou os níveis de ansiedade-traço competitiva em atletas infanto-juvenis do sexo masculino de diferentes modalidades e no qual os atletas de voleibol apresentaram níveis médios de ansiedade-traço competitiva ${ }^{15}$. Um estudo realizado com atletas de voleibol que comparou os níveis de ansiedade-traço segundo a idade e a modalidade esportiva também obteve níveis médios de ansiedade-traço competitiva ${ }^{23}$.

Uma pesquisa realizada para investigar os níveis de ansiedade-traço competitiva e compará-la entre as modalidades esportivas encontrou níveis médios em atletas de voleibol ${ }^{20}$, o que também concorda com os resultados encontrados no presente estudo.

Outro estudo que contribuiu para a discussão desses dados foi a pesquisa que verificou os níveis de ansiedade em atletas escolares de voleibol de ambos os sexos, com idades entre 15 e 17 anos, a qual constatou que a média do grupo é de níveis médios de ansiedade ${ }^{13}$. Com base nos estudos apresentados e nos resultados encontrados, pode-se dizer de uma forma geral que atletas de voleibol apresentam um nível médio de ansiedade-traço competitiva.

Em se tratando da ansiedade-estado, sabe-se que, durante competiçóes de esportes que exigem concentração, agilidade e estratégias, os níveis de ansiedade cognitiva devem estar em menores escalas, pois está relacionada com os pensamentos do indivíduo acerca de sua atuação na partida. Esses pensamentos podem ser duvidosos a respeito de seu desempenho e causar medo, apreensão ou apatia, produzindo efeitos negativos sobre o atleta ${ }^{24}$.

Quanto à ansiedade somática, é sabido que, durante competiçóes de modalidades esportivas que exigem rapidez, resistência física e força, ela deve estar em níveis mais elevados do que em uma situação normal, visto que diz respeito à ativação fisiológica e que leva a pessoa a um estado de alerta.
Já quanto à autoconfiança, caracterizada como a confiança que o atleta tem no seu rendimento, pode-se afirmar que níveis mais elevados seriam melhores para o desempenho durante uma competição ${ }^{24}$.

Para ansiedade cognitiva, os resultados encontrados na categoria infantil masculino e infanto-juvenil feminino corroboram o estudo que buscou investigar o nível de ansiedade pré-competitiva em atletas de modalidades individuais e coletivas e verificar a diferença do nível de ansiedade pré-competitiva nas diferentes modalidades, o qual observou que os níveis de ansiedade cognitiva são baixos nos esportes coletivos 5 . Esse achado contribuiu para os achados do presente estudo, pois apenas a categoria infantil feminino apresentou níveis moderados de ansiedade cognitiva.

Um estudo realizado com nadadores amadores do sexo masculino e feminino com média de idade de 17,8 $\pm 2,8$ anos buscou verificar os níveis de ansiedade-traço e estado nesses atletas e verificou que $56 \%$ da amostra apresentou níveis moderados de ansiedade cognitiva e apenas $31,37 \%$ apresentou nível baixo ${ }^{17}$.

Os achados quanto à ansiedade cognitiva podem ser explicados pelo fato de a primeira etapa da Liga de Voleibol do Paraná ter sido disputada em Guarapuava, o que contribuiu para que os atletas se sentissem mais à vontade com a situaçáa. Uma das explicativas para tanto seria que os níveis de ansiedade tendem a aumentar quando os jogos são disputados "fora de casa"; de fato, verifica-se que o benefício de jogar "em casa" tem sido um fator determinante dos resultados finais nas diferentes modalidades esportivas 5 .

No que se refere à ansiedade somática, os resultados expostos no presente estudo corroboram o estudo que encontrou níveis baixos desse tipo de ansiedade na sua amostra, em que as mulheres apresentaram níveis mais elevados que os homens ${ }^{17}$.

Observaram-se, no presente estudo, valores significativos nas categorias femininas, sendo que a infanto-juvenil apresentou níveis menos elevados do que a infantil. Isso pode ser justificado pelo fato de que atletas com mais experiência em competiçóes, caso da categoria infanto-juvenil, apresentam menores níveis somáticos ${ }^{17}$.

Diferentes níveis para ansiedade somática foram encontrados em pesquisa realizada para investigar os níveis de ansiedade pré-competitiva e autoconfiança em atletas de hóquei em patins. Esse estudo verificou que os atletas apresentaram níveis médios de ansiedade somática, mesmo jogando em casa ${ }^{25}$, diferentemente dos achados do presente estudo, em que os atletas apresentaram baixos níveis de ansiedade somática jogando em casa.

Já uma pesquisa realizada com atletas de polo aquático para avaliar a ansiedade e as habilidades psicológicas desses indivíduos observou que os níveis de ansiedade 
somática se encontram baixos ${ }^{26}$, em concordância com os valores obtidos nesta pesquisa.

Quanto ao terceiro componente da ansiedade-estado, a autoconfiança, os níveis se encontram elevados. No mesmo sentido, pesquisa realizada para verificar a ansiedade e associá-la ao desempenho motor de atletas de golfe constatou que os níveis de autoconfiança eram elevados e os de ansiedade cognitiva e somática, bai$\operatorname{xos}^{16}$, contribuindo com o presente estudo.

$\mathrm{O}$ estudo que analisou as diferenças no tipo de ansiedade entre jogadores titulares e reservas de futsal também constatou que a média de autoconfiança em todos os atletas apresentou-se alta ${ }^{24}$.

Todos os resultados para ansiedade-estado complementam os estudos da área. Ressalta-se que a autoconfiança tem relação direta com a ansiedade cognitiva e somática ${ }^{27}$, uma vez que os níveis de ansiedade cognitiva e somática se apresentaram, na maioria da amostra, baixos e moderados.

\section{Conclusão}

Ao considerar os objetivos iniciais do presente estudo, que eram avaliar os níveis de ansiedade-traço e estado e verificar se havia diferença entre as categorias e entre os gêneros, os resultados encontrados mostram que, de modo geral, não há diferença, uma vez que observou-se diferença estatisticamente significativa apenas entre a categoria infantil feminino e a categoria infantojuvenil para níveis de ansiedade somática.

Todas as categorias apresentaram níveis baixos para ansiedade cognitiva. Isso mostra que os atletas se mostram seguros com a competição. Uma possível explicação seria a de que estavam jogando em casa e era o primeiro jogo de todas as equipes. Esse fato pode ter diminuído a preocupaçáo com o resultado da partida, pois os atletas não tinham a "obrigação" de vencer.

Já para ansiedade somática, os valores foram baixos, demonstrando pouca ativação antes da competiçãa. No que se refere à autoconfiança, os resultados foram elevados, contribuindo para a ideia de que a autoconfiança tem relaçáo direta com a ansiedade cognitiva. A categoria que apresentou níveis mais elevados de autoconfiança foi a categoria infanto-juvenil; é possível que isso se deva ao fato de que essa categoria é mais experiente, uma vez que compete há mais tempo.

No entanto, são necessários mais estudos nessa área para investigar os níveis de ansiedade nas diferentes modalidades, entre diferentes gêneros e com relação à experiência obtida em cada modalidade e, assim, identificar diferenças significativas entre esses fatores.

\section{Referências}

1. Silveira RO. Análise dos níveis de ansiedade pré-competitiva de atletas de futsal [Monografia]. Belo Horizonte: Universidade Federal de Minas Gerais; 2009.

2. Weinberg R, Gould D. Fundamentos da psicologia do esporte e do exercício. São Paulo: Artmed; 2001.

3. Mosqueira J, Stobaus C. Psicologia do desporto. Porto Alegre: Edufrgs; 1984.

4. Davidoff LL. Introduçấo à psicologia. $3^{a}$ ed. São Paulo: Makron Books; 2001.

5. Alexandre B. Ansiedade pré-competitiva em modalidades de esportes coletivos e individuais [Monografia]. Criciúma: Universidade do Extremo Sul Catarinense; 2010.

6. Cruz J. Stress e ansiedade na competição desportiva: natureza, efeitos e avaliaçáo. In: Cruz J, editor. Manual de psicologia do desporto. Universidade do Minho: Instituto de Educação e Psicologia; 1996.

7. Samulski DM. Psicologia do esporte. São Paulo: Manole; 2002.

8. Martens R, Vealley R, Burton D. Competitive anxiety in sport. Champaign (IL): Human Kinetics Publishers; 1990.

9. Machado AA. Psicologia do esporte: da educação física escolar ao treinamento esportivo. Sáo Paulo: Guanabara Koogan; 2006.

10. Sonoo CN, et al. Ansiedade e desempenho: um estudo com uma equipe infantil de voleibol feminino. Motriz. $2010 \mathrm{Jul} /$ Set;16(3):629-37.

11. Becker Junior B. Os níveis de ansiedade do tenista. Match Point. 1989;2(25):10.

12. Cratty BJ. Psicologia no esporte. $2^{\text {a }}$ ed. Rio de janeiro: Prentice-Hall do Brasil; 1984.

13. Aplewicz JFG, et al. Níveis de ansiedade em atletas escolares de voleibol de faixa etária de 15 a 17 anos. Rev CPAQV. 2009; 1(1).

14. Ferreira JS, Leite LPR, Nascimento CMC. Relação entre níveis de ansiedade-traço competitiva e idade de atletas de voleibol e análise destes níveis pré e pós- competição. Motriz. 2010 Out/Dez;16(4):853-7.

15. Interdonato GC, et al. Análise da ansiedade-traço competitiva em jovens atletas. Conexóes. 2010 Set/Dez;8(3):1-9.

16. Martins NF. Associação entre ansiedade e desempenho motor em atletas de golfe da cidade de Maringá [Monografia]. Maringá: Universidade Estadual de Maringá; 2010.

17. Souza MAP, Teixeira RB, Lobato PL. Manifestação da ansiedade pré-competitiva em nadadores amadores. Rev Educ Fís/UEM. 2012;23(2):195-203.

18. Martens R. Sport Competition Anxiety. 2a ed. Champaign (IL): Human Kinetics Publishers; 1977.

19. Severo AL. Níveis de ansiedade-traço e ansiedade-estado em nadadores juvenis de ambos os sexos do estado do Paraná [Dissertação]. Santa Maria: Universidade Federal de Santa Maria; 1994. 
20. Bertuol L, Valentini NC. Ansiedade competitiva de adolescentes: gênero, maturação, nível de experiência e modalidades esportivas. Rev Educ Fís/UEM 2006;17(1):65-74.

21. Machado AA. Interferência da torcida na ansiedade e agressividade de atletas adolescentes [Tese]. Rio Claro: Universidade Estadual Paulista; 1998.

22. Gonçalvez MP, Belo RP. Ansiedade-traço competitiva: diferenças quanto ao gênero, faixa etária, experiência em competiçóes e modalidade esportiva em jovens atletas. PsicoUSF. 2007 Jul/Dez;12(2):301-7.

23. Santos SG, Pereira AS. Perfil do nível de ansiedade-traço pré-competitiva de atletas de esportes coletivos e individuais do estado do Paraná. Movimento. 1997;IV(6):3-13.
24. Bochini D, et al. Análise dos tipos de ansiedade entre jogadores titulares e reservas de futsal. Conexóes. 2008 Jul;6(ed. esp.):522-32.

25. Botelho M, Ferreira EMRS. O estado de ansiedade précompetitiva e autoconfiança: estudo realizado com jogadores seniores de hóquei em patins [Monografia]. Porto: Faculdade de Desporto, Universidade do Porto; 2006.

26. Penas DNDF. Ansiedade e habilidades psicológicas em atletas de polo aquático [Monografia]. Coimbra: Faculdade de Ciências do Desporto e Educação Física, Universidade de Coimbra; 2005.

27. Barbacena MM, Grisi RNF. Nível de ansiedade pré-competitiva em atletas de natação. Rev Facul Educ Fís Unicamp. 2008;6(1):31-9. 